\title{
Modeling the dichroic absorption band edge and light-induced magnetism in EuTe
}

\author{
A. B. Henriques and M. A. Manfrini \\ Instituto de Física, Universidade de São Paulo, Caixa Postale 66318, 05315-970 São Paulo, Brazil \\ P. H. O. Rappl and E. Abramof \\ LAS-INPE, Avenida dos Astronautas, 1758-Jd. Granja, 12227-010 São José dos Campos, Brazil \\ (Received 26 March 2007; revised manuscript received 15 July 2007; published 11 January 2008)
}

\begin{abstract}
The band-edge optical absorption in EuTe is studied in the framework of the $5 d$ conduction band atomic model. Both relaxed antiferromagnetic order, and ferromagnetic order induced by an external magnetic field, were analyzed. For ferromagnetic arrangement, the absorption is characterized by a hugely dichroic doublet of narrow lines. In the antiferromagnetic order, the spectrum is blueshifted, becomes much broader and weaker, and dichroism is suppressed. These results are in excellent qualitative and quantitative agreement with experimental observations on EuTe and EuSe published by us previously [Phys. Rev. B 72, 155337 (2005)]. The possibility of inducing ferromagnetic order by illuminating the material at photon energies resonant with the band gap is also discussed.
\end{abstract}

DOI: $10.1103 /$ PhysRevB.77.035204

PACS number(s): 78.20.-e

\section{INTRODUCTION}

Europium chalcogenides $(\mathrm{Eu} X)$ have for a long time attracted interest because of the huge magnetic moment associated with the lattice sites occupied by europium. It was early envisaged that a high density of magnetic moments in a crystal transparent in the visible range could be used for the construction of unique magneto-optical devices. ${ }^{1}$ The drawback, however, is the low critical temperature above which magnetic order is destroyed in $\mathrm{Eu} X$, and effort has been employed to raise their critical temperatures by introducing dopants $^{2}$ or mechanical strain. ${ }^{3}$ The interest in $\mathrm{EuX}$ was invigorated with the advent of spintronics, whose implementation depends on materials in which it is possible to control the electronic spin and the ferromagnetism externally. ${ }^{4}$ Spintronic devices require a source of spin-polarized electrical current, ${ }^{5}$ and $\mathrm{Eu} X$ is considered to be a candidate material for spin filtering. ${ }^{6}$ Using ferromagnetic EuO quantum structures, it is possible to produce spin-polarized currents reaching $100 \%,{ }^{7}$ which is larger than the theoretical limit for conventional semiconductor quantum structures. ${ }^{8}$ Light-induced magnetic order ${ }^{9}$ in materials is also an interesting effect for spintronic applications, ${ }^{10,11}$ which has not yet been explored in $\mathrm{Eu} X$.

To foresee potential applications of $\mathrm{Eu} X$ an understanding of their electronic band structure is required. However, basic open questions concerning the nature of their band-edge energy structure still remain. Recently, narrow lines in the band-edge absorption spectra of EuTe and EuSe in the ferromagnetic order were discovered ${ }^{12}$ (the ferromagnetic order was induced by an external field). These lines showed a huge magnetic circular dichroism. It is not yet possible to explain these observations on the basis of first-principle numerical ( $a b$ initio) modeling of $\mathrm{Eu} X$, since the results of this type of calculations are not accurate enough, because of the complexity of $\mathrm{Eu}$, which is a heavy atom with many electrons in internal cores. On the other hand, much insight into the band structure can be gained by using so-called semiempirical atomic analytical models, which are based on parameters ob- tained from experimental observations for these materials. ${ }^{13}$ Such models provide the basis for the present understanding of the main $\mathrm{Eu} X$ magneto-optical characteristics. ${ }^{14,15}$ In the $5 d$ conduction band model, the band-edge absorption is associated with electronic transitions from a strongly localized $4 f^{7}$ state to a narrow conduction band state built from $5 d$ atomic orbitals of the $\mathrm{Eu}^{2+}$, split by the crystal field.

Various energy level schemes have been attempted to describe the band-edge electronic structure in $\mathrm{EuX} .{ }^{16-19} \mathrm{Al}-$ though the main gross magneto-optical properties could be described, a description of the band-edge absorption failed (see discussion in Ref. 15, Sec. 3.2.2, Ref. 16, Sec. D, and Ref. 17, Sec. I.5). Although an old problem, it has not been resolved to this day, and it is the subject of the present paper. In our previous investigation we showed that the $5 d$ conduction band model could explain very well the sharp and dichroic absorption lines seen at high magnetic fields, when the arrangement of the $\mathrm{Eu}^{2+}$ was ferromagnetic. ${ }^{12}$ However, it remained unclear why the narrow absorption lines, seen in high magnetic fields, disappeared almost completely when the magnetic field was removed, and the absorption coefficient decreased. It was speculated that the large dependence of the oscillator strength on the magnetic field could be associated with electron-hole interaction effects; ${ }^{12}$ however, subsequent analysis showed that this hypothesis was unlikely. ${ }^{20,21}$

In this paper, we show that the $\left|4 f^{7}\left({ }^{8} S_{7 / 2}\right)\right\rangle$ $\rightarrow\left|4 f^{6}\left({ }^{7} F\right) J M_{J} ; X \sigma\right\rangle$ energy level scheme used in Ref. 12 to describe the absorption edge in high magnetic fields can also describe accurately the zero field spectrum, if the model is extended to incorporate the multiple antiferromagnetic domain arrangement of the $\mathrm{Eu}^{2+}$ spins that occurs at $B=0 .{ }^{14}$ The incorporation of an antiferromagnetic (AF) domain structure leads to a broad absorption spectrum with no resolved features, in agreement with experiment. It is shown that the extended model provides a faithful quantitative description of absorption both at high and zero magnetic fields. Furthermore, as a by-product, the extended model allows for the calculation of the dependence of the absorption spectrum 
on the direction of light travel in respect to the crystalline directions, and it is found that only a weak absorption anisotropy exists. The results allow us to speculate on the possibility of inducing ferromagnetic order in $\mathrm{Eu} X$ by illuminating the sample with light resonant with the band-edge absorption. It is predicted that, due to the $d-f$ exchange interaction, a photoexcited electron can induce a robust ferromagnetic alignment of the $\mathrm{Eu}^{2+}$ spins in a region whose dimension has a characteristic radius of $R \sim 10 \AA$.

\section{MODEL}

It is well-accepted that the $4 f^{7}$ electrons of the $\mathrm{Eu}^{2+}$ ions in the $\mathrm{EuX}$ crystal lattice are the highest energy electronic levels occupied at $T=0 \mathrm{~K} \cdot{ }^{14,15}$ Hence, from Hund's rule, the valence band in $\mathrm{EuX}$ is described by a localized state $4 f^{7}\left({ }^{8} S_{7 / 2}\right)$. The band-edge absorption of $\mathrm{Eu} X$ is then described by electronic transitions from the $4 f^{7}\left({ }^{8} S_{7 / 2}\right)$ to the lowest energy conduction band. It must be taken into account that when an electron is removed from the $4 f^{7}\left({ }^{8} S_{7 / 2}\right)$ state, a strong spin-orbit interaction arises between total orbital and spin momenta of the six remaining core electrons, and consequently the core can be left behind in one of seven different states, namely, the $4 f^{6}\left({ }^{7} F\right) J M_{J}$, where $J=0, \ldots, 6$ states. According to the Landé interval rule, the energy of these core states is given by $E_{J}=\frac{1}{2} J(J+1) \lambda_{4 f}$, where $\lambda_{4 f}$ is the spinorbit energy constant for the $4 f^{6}$ electrons.

To describe the lowest energy conduction band, the $5 d$ conduction band model has been proposed. ${ }^{16}$ In this model, the $5 d$ orbitals of the $\mathrm{Eu}^{2+}$ ions, split by the crystal field and resonantly coupled throughout the crystal lattice, give rise to a narrow conduction band. EuX has an $f c c$ crystal structure, hence the $\mathrm{Eu}^{2+}$ ions are located in a crystal field of octahedral symmetry. The $5 d$ level splits into a low energy $t_{2 g}$ threefold degenerate energy level (described by orbitals $d_{x y}$, $d_{y z}$, and $d_{z x}$ ), and a high energy $e_{g}$ twofold degenerate energy level (described by orbitals $d_{z^{2}}$ and $d_{x^{2}-y^{2}}$ ). The splitting between the $t_{2 g}$ and the $e_{g}$ manifolds is estimated to be greater than $1 \mathrm{eV},{ }^{24,15}$ hence for the band-edge absorption spectrum the $e_{g}$ states can be ignored. The $5 d\left(t_{2 g}\right)$ state is further split by the spin-orbit interaction; ${ }^{12,16}$ however, previous investigations have indicated that this splitting has only a minor influence on the spectrum. ${ }^{12,22}$ This is explained by the broadening of the atomic energy levels into a conduction energy band, which washes out the fine structure in the $t_{2 g}$ manifold, and for simplicity this spin-orbit interaction is ignored in the present analysis.

In the framework of the level scheme described, the contribution stemming from the $5 d$ conduction band to the bandedge absorption is connected to electronic transitions $\left|4 f^{7}\left({ }^{8} S_{7 / 2}\right)\right\rangle \rightarrow\left|4 f^{6}\left({ }^{7} F\right) J M_{J} ; X \sigma\right\rangle$, where $X$ represents one of the three orbital states in the $5 d\left(t_{2 g}\right)$ subset $\left(d_{x y}, d_{y z}\right.$, or $\left.d_{z x}\right)$, and $\sigma$ represents the spin state of the electron in the $X$ state. The electric dipole matrix element, associated with such an electronic transition, for circularly polarized light, is given by

$$
\begin{aligned}
\mu^{ \pm}\left(J, M_{J}, X\right)= & \left\langle 4 f^{7}\left({ }^{8} S_{7 / 2}\right)\left|\sum_{k=1}^{7}\left(x_{k} \pm i y_{k}\right)\right| 4 f^{6}\left({ }^{7} F\right) J M_{J} ; X \sigma\right\rangle \\
= & \sum_{M_{L} M_{S}}\left({ }^{7} F M_{L} M_{S} \mid J M_{J}\right)\left\langle 4 f^{7}\left({ }^{8} S_{7 / 2}\right)\right| \sum_{k=1}^{7}\left(x_{k} \pm i y_{k}\right) \\
& \times\left|4 f^{6}\left({ }^{7} F M_{L} M_{S}\right) ; X \sigma\right\rangle \\
= & {\left[{ }^{7} F M_{L}=\left(M_{J}-3\right) M_{S}=3 \mid J M_{J}\right] } \\
& \times\left\langle 4 f^{7}\left({ }^{8} S_{7 / 2}\right)\right| \sum_{k=1}^{7}\left(x_{k} \pm i y_{k}\right) \\
& \times\left|4 f^{6}\left[{ }^{7} F M_{L}=\left(M_{J}-3\right) M_{S}=3\right] ; X \uparrow\right\rangle
\end{aligned}
$$

where the $\left({ }^{7} F\right) J M_{J}$ states were expanded in terms of $\left({ }^{7} F\right) M_{L} M_{S}$ states using Clebsh-Gordan coefficients $\left(L S M_{L} M_{S} \mid J M_{J}\right)$, the spin conservation in an electric-dipole transition was used $(|X \uparrow\rangle$ represents an electron in an $X$ state whose spin is parallel to the direction of the $\mathrm{Eu}^{2+}$ spin where the excitation occurred), the property $\left(L S M_{L} M_{S} \mid J M_{J}\right)=0$ unless $M_{J}=M_{L}+M_{S}$ was used, $x_{k}, y_{k}$ represent the coordinates of the $k$ th electron perpendicular to the direction of light propagation, and the plus (minus) superscript holds for right-hand (left-hand) circularly polarized light. The summation over $k$ can now be done by noting that the many-electron matrix element on the right-hand side of Eq. (1) is equivalent to a single electron matrix element, namely one that connects a $4 f$ orbital, with $m=-M_{J}+3$, and an $X$ orbital. Therefore Eq. (1) can be rewritten as

$$
\begin{aligned}
\mu^{ \pm}\left(J, M_{J}, X\right)= & {\left[{ }^{7} F M_{L}=\left(M_{J}-3\right) M_{S}=3 \mid J M_{J}\right] } \\
& \times\left\langle 4 f\left(m=-M_{J}+3\right)|x \pm i y| X\right\rangle .
\end{aligned}
$$

To calculate the matrix element $\langle 4 f m|z| X\rangle$, the wave functions must be brought to the same reference frame that is used to describe the propagating light. This can be done using Wigner rotation matrices $D_{m m^{\prime}}^{(j)}(\alpha, \beta, \gamma)$ (explicit forms of these matrices are given in Ref. 23). For instance,

$$
|4 f m\rangle=\sum_{m_{1}=-3}^{+3} D_{m m_{1}}^{(3)}\left(\alpha_{S}, \beta_{S}, \gamma_{S}\right)\left|4 f m_{1}\right\rangle,
$$

where $\alpha_{S}, \beta_{S}$, and $\gamma_{S}$ are the Euler angles that transform the reference frame used to describe the $|4 \mathrm{fm}\rangle$ state (in which the $z$ direction is the angular momentum quantization axis for the ${ }^{8} S_{7 / 2}$ state) into the reference frame for light propagation (in which light travels along $z$ ). Similarly, since the $X$ states are linear combinations of $\left|5 d m^{\prime}\right\rangle$ orbitals, i.e., $X$ $=\Sigma B\left(X, m^{\prime}\right)\left|5 d m^{\prime}\right\rangle$ [for instance, if $X=d_{x y}$, then $B(X, 2)$ $=-B(X,-2)=\frac{1}{\sqrt{2}}$, all other $\left.B\left(X, m^{\prime}\right)=0\right]$,

$$
X=\sum_{m^{\prime}=-2}^{+2} B\left(X, m^{\prime}\right) \sum_{m_{2}=-2}^{+2} D_{m^{\prime} m_{2}}^{(2)}\left(\alpha_{c}, \beta_{c}, \gamma_{c}\right)\left|5 d m_{2}\right\rangle,
$$

where $\alpha_{c}, \beta_{c}$, and $\gamma_{c}$ are the Euler angles that transform the reference frame of the crystal structure (in which the $z$ direction is parallel to the crystal direction of Miller indices [001]) into the frame for light propagation. (For example, if 
light travels along [111], and the $\mathrm{Eu}^{2+}$ spins are oriented

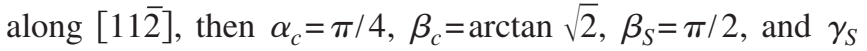
$=\pi$, while $\gamma_{c}$ and $\alpha_{S}$ can take arbitrary values because only the $z$ direction is strictly defined in the ionic spin and photon reference frames.) With the substitutions of Eqs. (3) and (4) in Eq. (2), the dipole matrix elements can be calculated for any direction of light in respect to the crystalline axes, thus for a given direction of quantization of the $\mathrm{Eu}^{2+}$ spins in the crystal,

$$
\begin{aligned}
\mu^{ \pm}\left(J, M_{J}, X\right)= & {\left[{ }^{7} F M_{L}=\left(M_{J}-3\right) M_{S}=3 \mid J M_{J}\right] } \\
& \times \sum_{m_{1}=-3}^{m_{1}=+3} D_{-M_{J}+3, m_{1}}^{(3)^{*}}\left(\alpha_{S}, \beta_{S}, \gamma_{S}\right) \\
& \times \sum_{m^{\prime}=-2}^{+2} B\left(X, m^{\prime}\right) \sum_{m_{2}=-2}^{m_{2}=+2} D_{m^{\prime} m_{2}}^{(2)}\left(\alpha_{c}, \beta_{c}, \gamma_{c}\right) \mathcal{M}_{m_{1} m_{2}}^{ \pm},
\end{aligned}
$$

where the matrices $\mathcal{M}_{m_{1} m_{2}}^{ \pm}=\left\langle 4 f m_{1}|x \pm i y| 5 d m_{2}\right\rangle \quad\left(m_{1}\right.$ $=-3, \ldots,+3$ and $\left.m_{2}=-2, \ldots,+2\right)$ are obtained using the explicit form ${ }^{24}$ of the angular part of the atomic $|4 f m\rangle$ and $|5 d m\rangle$ wave functions,

$$
\mathcal{M}_{p q}^{+}=i r_{d f}\left[\begin{array}{c|ccccc}
p & +2 & +1 & 0 & -1 & -2 \\
\hline+3 & \sqrt{\frac{6}{7}} & 0 & 0 & 0 & 0 \\
+2 & 0 & \frac{2}{\sqrt{7}} & 0 & 0 & 0 \\
0 & 0 & \sqrt{\frac{12}{35}} & 0 & 0 \\
-1 & 0 & 0 & 0 & \sqrt{\frac{6}{35}} & 0 \\
-2 & 0 & 0 & 0 & 0 & 0 \\
-3 & 0 & 0 & 0 & 0 & 0
\end{array}\right] .
$$

In Eq. (6), $r_{d f}=\int \mathcal{R}_{4 f}(r) \mathcal{R}_{5 d}(r) r^{3} d r$ is the $4 f-5 d$ radial integral, which is treated as an adjustable parameter, due to the deformation of the atomic orbitals by the crystal field. For the left-hand circularly polarized light dipole matrix element, the result is $\mathcal{M}_{p q}^{-}=\left[\mathcal{M}_{-p,-q}^{+}\right]^{*}$.

The change in the energy of the electronic system in the transition is given by $\Delta E=E_{J}+E_{X}$, where $E_{X}$ is the energy of the electron in the final state $X$. Using the dipole matrix element (5), the band-edge absorption coefficient, at a photon energy $\hbar \omega$, will be given by

$$
\begin{aligned}
\alpha^{ \pm}(\hbar \omega)= & \frac{N \pi e^{2} \hbar \omega}{2 c \epsilon_{0} \hbar} \sum_{J=0}^{6} \sum_{M_{J}=-J}^{+J} \sum_{X}\left|\mu^{ \pm}\left(J, M_{J}, X\right)\right|^{2} \\
& \times \delta\left(E_{X}+E_{J}-\hbar \omega\right),
\end{aligned}
$$

where $N=4 / a^{3}$ is the volume density of $\mathrm{Eu}^{2+}$ ions, and $a$ $=6.600 \AA$ is the EuTe lattice parameter.

To complete the picture, the magnetic order of the lattice must be taken into account. At zero external magnetic field, below the Néel temperature, the order of the spins is antiferromagnetic. The $\mathrm{Eu}^{2+}$ spins lie in the $\{111\}$ planes, and since there are four equivalent such planes, a sample of EuTe will consist of four groups of domains (so-called $T$ domains), each associated with a particular $\{111\}$ plane in which the spins lie. Additionally, within each $T$ domain, the spins are aligned along one of three $\langle 11 \overline{2}\rangle$ equivalent directions, hence each $T$ domain contains three so-called $S$ domains, in which the spins are oriented along different $\langle 11 \overline{2}\rangle$ directions. ${ }^{14}$ For a given direction of light travel, there will be altogether 24 possible $\mathrm{Eu}^{2+}$ spin orientations in the path of light, which are assumed to occur with equal probability. The absorption spectrum will then be given by Eq. (7), averaged over all these 24 different orientations:

$$
\begin{aligned}
\alpha^{ \pm}(\hbar \omega)= & \frac{N \pi e^{2} \hbar \omega}{2 c \epsilon_{0} \hbar} \sum_{J=0}^{6} \sum_{M_{J}=-J}^{+J} \sum_{X} \overline{\left|\mu^{ \pm}\left(J, M_{J}, X\right)\right|^{2}} \\
& \times \delta\left(E_{G}+E_{J}-\hbar \omega\right),
\end{aligned}
$$

where $E_{G}=E_{X}$ represents the gap energy, the minimum energy transition at zero magnetic field.

The situation becomes simpler when the sample is submitted to an external magnetic field, strong enough to align all $\mathrm{Eu}^{2+}$ spins. Under this condition, due to the $d-f$ exchange interaction, the energy of the electrons in the $|X\rangle$ state is decreased by $J_{d f} S \sim 150 \mathrm{meV},{ }^{25}$ relative to their energy when the order of the spins in the lattice is antiferromagnetic. Therefore in the high magnetic field limit the absorption spectrum will be given by

$$
\begin{aligned}
\alpha^{ \pm}(\hbar \omega)= & \frac{N \pi e^{2} \hbar \omega}{2 c \epsilon_{0} \hbar} \sum_{J=0}^{6} \sum_{M_{J}=-J}^{+J} \sum_{X}\left|\mu^{ \pm}\left(J, M_{J}, X\right)\right|^{2} \\
& \times \delta\left(E_{G}-J_{d f} S+E_{J}-\hbar \omega\right) .
\end{aligned}
$$

Equations (8) and (9) are of course approximations. Observed spectral absorption lines are not infinitely sharp but possess a finite width, and the excited state $|X\rangle$ is not a strictly discrete energy level, but rather a state within a narrow conduction band that decays with a characteristic lifetime back to the original lower lying available levels. To bring the theory closer to the experimental situation, Dirac's $\delta$-function line shape in Eq. (7) was substituted by a symmetric Voigt function, built from a Lorentzian and a Gaussian of equal full widths at half maximum (FWHM). The FWHM of the Voigt function was fixed at $65 \mathrm{meV}$, which gives a good description of the absorption onset, as previous experiments on EuTe and EuSe have indicated. ${ }^{12,21}$ Parameter FWHM is in fact a measure of the energy width of the $5 d$ conduction band: since the $4 f^{7}\left({ }^{8} S_{7 / 2}\right)$ state is strongly 

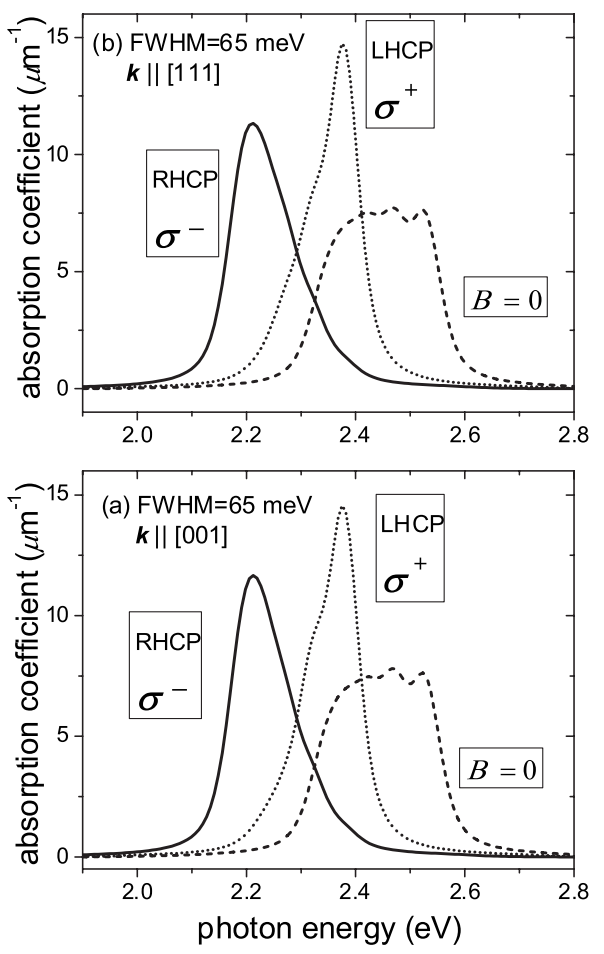

FIG. 1. Theoretical absorption of circularly polarized light in EuTe, in the Faraday configuration. Miller indices indicate the direction of light travel. Curves labeled $\sigma^{ \pm}$correspond to the absorption of photons that possess an angular momentum with projection $\pm \hbar$ in the direction of the $\mathrm{Eu}^{2+}$ spins, when $B$ is large and imposes ferromagnetic arrangement.

spatially localized in the $\mathrm{Eu}^{2+}$ ion, optical transitions to any $k$ point of the Brillouin zone of the $5 d$ extended states can take place. As a result, the absorption coefficient, associated with a fixed final state of the $\mathrm{Eu}^{3+}$ core, will be approximately proportional to the density of states of the $5 d$ conduction band.

\section{RESULTS AND DISCUSSION}

Figures 1(a) and 1(b) show the calculated band-edge absorption spectra of circularly polarized light of EuTe in the Faraday configuration, using a Voigt broadening function of $\mathrm{FWHM}=65 \mathrm{meV}$, for light propagating along the crystalline directions [001] and [111], using Eqs. (8) and (9). The parameters entering these equations were fixed at values: $J_{d f} S$ $=150 \mathrm{meV}$ and $E_{G}=2.321 \mathrm{eV}$, obtained from the best-fit analysis of the redshift of the absorption when a magnetic field is applied, ${ }^{25}$ and $\lambda_{4 f}=9.6 \mathrm{meV}, r_{d f}=0.18 \AA$, obtained from the line shape of the absorption spectrum under induced ferromagnetic order. ${ }^{12}$ Spectra shown in Fig. 1 are labeled as RHCP or LHCP, meaning that the circularly polarized photons carry an angular momentum whose projection is $+\hbar$ or $-\hbar$, along the direction of light travel, respectively.

When the sample is submitted to a magnetic field that aligns the $\mathrm{Eu}^{2+}$ spins antiparallel to $B$, the direction of circular polarization can also be indicated by a label $\sigma^{ \pm}$, meaning that the component of photon angular momentum parallel to the direction of the applied magnetic field is $\pm \hbar$. As Figs. 1(a) and 1(b) show, the $\sigma^{+}$spectra taken either in the [001] or in the [111] direction, are almost identical and have the same characteristics, namely: (1) under a strong magnetic field, which imposes ferromagnetic ordering of the $\mathrm{Eu}^{2+}$ spins, absorption is strongly dichroic; (2) when the magnetic field is removed, the absorption spectrum blueshifts, becomes much broader, and the absorption coefficient becomes smaller; and (3) at $B=0$, dichroism is suppressed, as expected for an antiferromagnet, due to symmetry. These results are in remarkable agreement with published experimental measurements of the absorption spectra taken in the interval 1.8$2.5 \mathrm{eV} .{ }^{12,21}$ At energies higher than $2.5 \mathrm{eV}$, the experimental absorption increases monotonously, due to optical transitions to electronic states of higher energies. ${ }^{12}$ These states were not included in the theory, therefore our model is useful to describe only an energy interval of length $\sim 400 \mathrm{meV}$ at the absorption onset (the 2.0-2.4 eV interval in EuTe). For light traveling along other crystalline directions, our calculation shows that the absorption spectra for either ferromagnetic or antiferromagnetic order remains nearly the same as in Figs. 1(a) and 1(b), thus the absorption is almost isotropic. The theoretical model described in this paper can also be used to calculate the absorption spectrum for any magnetic field value and orientation, and a detailed comparison with experimental data for a range of magnetic fields is presented in Ref. 26.

An interesting question is whether light can be used to induce ferromagnetic order in EuTe, since the ability to externally control the magnetic properties of materials is desired for application in spintronic devices. ${ }^{10}$ If in the ground state the order of the $\mathrm{Eu}^{2+}$ spins is antiferromagnetic (at $B$ $=0$ ) or disordered (above the Néel temperature), once an electron is transferred optically to an extended $5 d\left(t_{2 g}\right)$ state, the excited electron can reduce its energy by aligning around itself the spins of the $\mathrm{Eu}^{2+}$ with its own, through the $d-f$ exchange interaction. The excited electron wave function would then be accompanied by a surrounding cloud of ferromagnetically aligned $\mathrm{Eu}^{2+}$ spins, forming a so-called magnetic polaron. By aligning all the $\mathrm{Eu}^{2+}$ spins in its neighborhood, the electron reduces its energy by $J_{d f} S=150 \mathrm{meV} .^{25}$ EuTe is an antiferromagnet of the second kind, ${ }^{27}$ therefore the energy required to flip an $\mathrm{Eu}^{2+}$ spin is $\delta U=12\left|J_{2}\right| S^{2}$, where $J_{2}=-1.30 \times 10^{-5} \mathrm{eV}$ (Ref. 14) is the second neighbor $\mathrm{Eu}^{2+}-\mathrm{Eu}^{2+}$ superexchange interaction constant, and $S=7 / 2$. The $\mathrm{Eu}^{2+}$ spin-flip energy cost favors spatial localization of the $5 d\left(t_{2 g}\right)$ electronic wave function in a finite volume. However, as the wave function is compressed, the stored kinetic energy increases, whose order of magnitude can be estimated from $K \sim \frac{\hbar^{2}}{2 m_{0} R^{2}}$. Thus the total energy change of the $5 d\left(t_{2 g}\right)$ wave function, for a radius of localization equal to $R$ within which the $\mathrm{Eu}^{2+}$ spins are flipped into a ferromagnetic arrangement, will be given by $\Delta E(R) \sim-J_{d f} S+K+\frac{4}{3} \pi R^{3} N \delta U$, where $N=\frac{4}{a^{3}}$ is the density of $\mathrm{Eu}^{2+}$ in the lattice. The minimum value of $\Delta E(R)$ occurs when $R \sim\left(\frac{\hbar^{2} a^{3}}{16 \pi m_{0} \delta U}\right)^{1 / 5} \sim 10 \AA$, which provides us with an order-of-magnitude estimate of the size of the magnetic polaron that could exist in EuTe. The magnetic moment of a single polaron can then be estimated, $\mu \sim \frac{4}{3} \pi R^{3} N g_{J} \mu_{B} \sqrt{J(J+1)} \sim 200 \mu_{B}$ where $g_{J}=2$ is the Landé 
gyromagnetic factor for $\mathrm{Eu}^{2+}, J=7 / 2$, and $\mu_{B}$ is Bohr's magneton. Such a light-induced magnetic moment could be easily detected using sensitive pump-probe optical techniques, pioneered over the last decade (see, for instance, Ref. 28). The estimated value $R \sim 10 \AA$ coincides with the radius of localization, which was previously estimated by an experimental study of the broadening of the band-edge $\sigma^{+}$absorption line in $\mathrm{Pb}_{x} \mathrm{Eu}_{1-x} \mathrm{Te}$, associated with stochastic alloy disorder. ${ }^{21,25}$

\section{CONCLUSIONS}

Light absorption was investigated in both relaxed (antiferromagnetic) EuTe and in EuTe submitted to a magnetic field large enough to impose ferromagnetic order. Only the contributions to the band-edge absorption coming from the $5 d$ conduction band was taken into account, and it is in outstanding agreement with experimental observations in an interval of photon energies of about $400 \mathrm{meV}$ above the onset of absorption. These results show that the band-edge absorption in $\mathrm{Eu} X$ can be fully described by the $\left|4 f^{7}\left({ }^{8} S_{7 / 2}\right)\right\rangle$ $\rightarrow\left|4 f^{6}\left({ }^{7} F\right) J M_{J} ; X \sigma\right\rangle$ energy level scheme, both in high magnetic fields when the spin arrangement is ferromagnetic, and in zero magnetic field when the sample is broken into a mul- tiple antiferromagnetic domain structure. Photons traveling in different crystalline directions were considered, and it is found that the absorption spectrum is nearly isotropic, with the same features. For ferromagnetic lattice order, the absorption spectrum is described by a doublet of circularly dichroic absorption lines (splitting $200 \mathrm{meV}$ ). For antiferromagnetic spin order, the absorption spectrum blueshifts and becomes weaker and broader, and circular dichroism is suppressed: these results can be traced to the formation of antiferromagnetic domains that are inherent to EuTe. Based on these results, one might predict that ferromagnetic order in the lattice, in the vicinity of the Eu site where the excitation occurred, could be induced by light, because of the strong $d-f$ exchange interaction, which will favor ferromagnetic alignment of the $\mathrm{Eu}^{2+}$ spins with the spin of the excited electron. Such light-induced magnetism is a prospective ingredient in spintronic devices. ${ }^{4}$ The results described for EuTe should be also valid for EuSe, whose band-edge absorption spectrum shows the same features as EuTe. ${ }^{12}$

\section{ACKNOWLEDGMENTS}

A.B.H. thanks V. Bindilatti for profitable discussions and acknowledges financial support provided by CNPq (Project No. 308116/2004-6).
${ }^{1}$ S. Methfessel, IEEE Trans. Magn. 1, 144 (1965).

${ }^{2}$ S. V. Molnar and M. W. Shafer, J. Appl. Phys. 41, 1093 (1970).

${ }^{3}$ R. T. Lechner, G. Springholz, T. U. Schulli, J. Stangl, T. Schwarzl, and G. Bauer, Phys. Rev. Lett. 94, 157201 (2005).

${ }^{4}$ I. Zutic, J. Fabian, and S. D. Sarma, Rev. Mod. Phys. 76, 323 (2004).

${ }^{5}$ S. Datta and B. Das, Appl. Phys. Lett. 56, 665 (1990).

${ }^{6}$ D. P. DiVincenzo, J. Appl. Phys. 85, 4785 (1999).

${ }^{7}$ T. S. Santos and J. S. Moodera, Phys. Rev. B 69, 241203(R) (2004).

${ }^{8}$ T. Koga, J. Nitta, H. Takayanagi, and S. Datta, Phys. Rev. Lett. 88, 126601 (2002).

${ }^{9}$ S. Markowski, T. Gurung, T. A. Nguyen, H. E. Jackson, G. K. L. M. Smith, and J. Kossut, Appl. Phys. Lett. 84, 3337 (2004).

${ }^{10}$ H. Ohno, D. Chiba, F. Matsukura, T. Omiya, E. Abe, T. Dietl, Y. Ohno, and K. Ohtani, Nature (London) 408, 944 (2000).

${ }^{11}$ A. H. MacDonald, P. Schiffer, and A. Samarth, Nat. Mater. 4, 195 (2005).

${ }^{12}$ A. B. Henriques, A. Wierts, M. A. Manfrini, G. Springholz, P. H. O. Rappl, E. Abramof, and A. Y. Ueta, Phys. Rev. B 72, 155337 (2005).

${ }^{13}$ T. Kasuya, J. Magn. Magn. Mater. 195, 141 (1999).

${ }^{14} \mathrm{P}$. Wachter, Handbook on the Physics and Chemistry of Rare Earths (North-Holland, Amsterdam, 1979), Vol. 1, p. 507.

${ }^{15}$ A. Mauger and C. Godart, Phys. Rep. 141, 51 (1986).

${ }^{16}$ G. Guntherodt, P. Wachter, and D. M. Imboden, Phys. Kondens. Mater. 12, 292 (1971).

${ }^{17}$ G. Guntherodt, P. Wachter, and D. M. Imboden, Phys. Condens.
Matter. 18, 37 (1974).

${ }^{18}$ O. Sakai, A. Yanase, and T. Kasuya, J. Phys. Soc. Jpn. 42, 596 (1977).

${ }^{19}$ W. Reim and J. Schoenes, Ferromagnetic Materials (NorthHolland, Amsterdam, 1990), Vol. 5, p. 183.

${ }^{20}$ A. B. Henriques, M. A. Manfrini, G. D. Galgano, B. L. Díaz, P. H. O. Rappl, and E. Abramof, Int. J. Mod. Phys. B 21, 1247 (2007).

${ }^{21}$ A. B. Henriques, G. D. Galgano, B. Díaz, P. H. O. Rappl, and E. Abramof, Proceedings of the 28th International Conference on the Physics of Semiconductors, AIP Conf. Proc. No. 893 (AIP, Melville, NY, 2007), p. 1233.

${ }^{22}$ C. R. Pidgeon, J. Feinleib, W. J. Scouler, J. Hanus, J. O. Dimmock, and T. B. Reed, Solid State Commun. 7, 1323 (1969).

${ }^{23}$ D. A. Varshalovich, A. N. Moskalev, and V. K. Khersonskii, Quantum Theory of Angular Momentum (World Scientific, Singapore, 1988).

${ }^{24}$ L. D. Landau and E. M. Lifshitz, Kvantovaya Mehanika (Nauka, Moscow, 1974).

${ }^{25}$ A. B. Henriques, L. K. Hanamoto, N. F. Oliveira, P. H. O. Rappl, E. Abramof, and A. Y. Ueta, J. Phys.: Condens. Matter 16, 5597 (2004).

${ }^{26}$ A. B. Henriques, G. D. Galgano, B. Díaz, P. H. O. Rappl, and E. Abramof, J. Phys.: Condens. Matter 19, 406234 (2007).

${ }^{27}$ J. S. Smart, Effective Field Theories of Magnetism (W. B. Saunders, London, 1966).

${ }^{28}$ Y. K. Kato, R. C. Myers, A. C. Gossard, and D. D. Awschalom, Science 306, 1910 (2004). 